PROCEEDINGS OF THE

AMERICAN MATHEMATICAL SOCIETY

Volume 129, Number 9, Pages 2541-2546

S 0002-9939(01)05853-1

Article electronically published on January 18, 2001

\title{
CONVEX POLYTOPES ALL OF WHOSE REVERSE LEXICOGRAPHIC INITIAL IDEALS ARE SQUAREFREE
}

\author{
HIDEFUMI OHSUGI AND TAKAYUKI HIBI
}

(Communicated by Wolmer V. Vasconcelos)

\begin{abstract}
A compressed polytope is an integral convex polytope any of whose reverse lexicographic initial ideals is squarefree. A sufficient condition for a $(0,1)$-polytope to be compressed will be presented. One of its immediate consequences is that the class of compressed $(0,1)$-polytopes includes (i) hypersimplices, (ii) order polytopes of finite partially ordered sets, and (iii) stable polytopes of perfect graphs.
\end{abstract}

\section{INTRODUCTION}

The triangulation of convex polytopes is one of the most traditional research areas in the theory of convex polytopes. A convex polytope $\mathcal{P} \subset \mathbb{R}^{n}$ is called integral if all vertices of $\mathcal{P}$ belong to $\mathbb{Z}^{n}$. Moreover, a convex polytope $\mathcal{P} \subset \mathbb{R}^{n}$ is said to be a $(0,1)$-polytope if each of its vertices belongs to $\{0,1\}^{n}$. Recall that a triangulation $\Delta$ of an integral convex polytope $\mathcal{P} \subset \mathbb{R}^{n}$ with $\mathcal{P} \cap \mathbb{Z}^{n}=\left\{\delta_{1}, \delta_{2}, \ldots\right\}$ is unimodular if the set of vertices $\left\{\alpha_{1}, \alpha_{2}, \ldots\right\}$ of each simplex belonging to $\Delta$ is a subset of $\mathcal{P} \cap \mathbb{Z}^{n}$ and satisfies the condition that $\left\{\left(\alpha_{1}, 1\right),\left(\alpha_{2}, 1\right), \ldots\right\} \subset \mathbb{Z}^{n+1}$ is part of a $\mathbb{Z}$-basis of the finitely generated abelian group $\left(\subset \mathbb{Z}^{n+1}\right)$ generated by $\left\{\left(\delta_{1}, 1\right),\left(\delta_{2}, 1\right), \ldots\right\}$. Many combinatorialists and algebraists have paid attention to unimodular triangulations of integral convex polytopes. For example, the resolution of singularities on toric varieties is equivalent to saying that, for any integral convex polytope $\mathcal{P}$, there exists an integer $N>0$ such that the integral convex polytope $N \mathcal{P}$ possesses a unimodular triangulation. On the other hand, if an integral convex polytope $\mathcal{P}$ possesses a unimodular triangulation, then the Ehrhart polynomial of $\mathcal{P}$ coincides with the Hilbert function of the Stanley-Reisner ring associated with the triangulation. From viewpoints of combinatorics, commutative algebra and algebraic geometry, the integral convex polytope with "many" unimodular triangulations is of great interest. An integral convex polytope is called unimodular if all of its triangulations are unimodular. A unimodular polytope enjoys many nice combinatorial properties. For example, it is known OhHerHi] that the Lawrence lifting of an integral convex polytope $\mathcal{P}$ is normal if and only if $\mathcal{P}$ is unimodular. A unimodular polytope is, however, quite rare. It seems to be reasonable to seek a natural class of integral convex polytopes with many unimodular triangulations.

Received by the editors November 3, 1999 and, in revised form, January 17, 2000.

2000 Mathematics Subject Classification. Primary 13P10, 52B20.

Key words and phrases. Compressed polytopes, initial ideals, unimodular triangulations.

The first author is supported by JSPS Research Fellowship for Young Scientists. 
One of the natural classes of such polytopes is introduced by Stanley Sta]. An integral convex polytope is called compressed if all of its "pulling triangulations" are unimodular. (An algebraic definition of compressed polytopes in terms of toric ideals will be given in the second paragraph.) In [Sta] it is shown that the convex polytope of all $n \times n$ doubly stochastic matrices is compressed. It is, however, still unclear if the class of compressed polytopes is enough large. Our original motivation to organize the present paper is, in order to understand that the class of compressed polytopes is rich in many distinguished polytopes, to find a sufficient condition for an integral convex polytope to be compressed.

Let $\mathcal{P} \subset \mathbb{R}^{n}$ be an integral convex polytope with the vertices $\delta_{1}, \delta_{2}, \ldots, \delta_{q}$ and $K\left[y_{1}, y_{2}, \ldots, y_{q}\right]$ the polynomial ring in $q$ variables over a field $K$. The toric ideal of $\mathcal{P}$ is the ideal $I_{\mathcal{P}} \subset K\left[y_{1}, y_{2}, \ldots, y_{q}\right]$ generated by all homogeneous binomials $f=\prod_{\ell=1}^{N} y_{t_{\ell}}-\prod_{\ell=1}^{N} y_{s_{\ell}}$, where $N=2,3, \ldots$, with $\sum_{\ell=1}^{N} \delta_{t_{\ell}}=\sum_{\ell=1}^{N} \delta_{s_{\ell}}$. (To avoid confusion we especially note that, in the present paper, to define the toric ideal of an integral convex polytope $\mathcal{P} \subset \mathbb{R}^{n}$ we use only the vertices of $\mathcal{P}$ and do not use $\mathcal{P} \cap \mathbb{Z}^{n}$. Thus, for example, the toric ideal of $\mathcal{P}$ coincides with that of $N \mathcal{P}$ for all integer $N>0$. If $\mathcal{P}$ is, e.g., a $(0,1)$-polytope, then $\mathcal{P} \cap \mathbb{Z}^{n}$ coincides with the set of vertices of $\mathcal{P}$.) A compressed polytope [Sta, p. 337] is an integral convex polytope $\mathcal{P} \subset \mathbb{R}^{n}$ such that the initial ideal of $I_{\mathcal{P}}$ with respect to any reverse lexicographic monomial order on $K\left[y_{1}, y_{2}, \ldots, y_{q}\right]$ is generated by squarefree monomials. (See [Stu, Corollary 8.9].) If $\mathcal{P} \subset \mathbb{R}^{n}$ is compressed, then all faces of $\mathcal{P}$ are again compressed.

As was stated in the first paragraph, it is known [Sta, Example 2.4 (b)] that the convex polytope of all $n \times n$ doubly stochastic matrices is compressed. Moreover, it is proved [Stu, Theorem 14.8] that all reverse lexicographic initial ideals of its toric ideal are generated by squarefree monomials of degree at most $n$. The purpose of the present paper is to discuss the technique appearing in the proof of [Stu], Theorem 14.8] in a much more general situation and to show that the convex polytope determined by a certain system of linear inequalities is compressed provided that the polytope is a $(0,1)$-polytope. More precisely,

Theorem. Let $a_{i j}, b_{i}$ and $\varepsilon_{i}, 1 \leq i \leq m, 1 \leq j \leq n$, be integers with each $\varepsilon_{i} \in\{0,1\}$. Suppose that the convex polytope $\mathcal{P} \subset \mathbb{R}^{n}$ consisting of all solutions $\left(x^{(1)}, x^{(2)}, \ldots, x^{(n)}\right)$ of the system of linear inequalities

$$
\begin{aligned}
b_{i} \leq \sum_{j=1}^{n} a_{i j} x^{(j)} \leq b_{i}+\varepsilon_{i}, & 1 \leq i \leq m, \\
0 \leq x^{(j)} \leq 1, & 1 \leq j \leq n,
\end{aligned}
$$

is a $(0,1)$-polytope. Then $\mathcal{P}$ is compressed.

Our situation is particularly nice if the coefficient matrix of the system of linear inequalities is totally unimodular, because the required assumption that the polytope is a $(0,1)$-polytope is automatically satisfied if the coefficient matrix is totally unimodular (Corollary 1.2). Example 1.3) says that the class of compressed $(0,1)$-polytopes includes (i) hypersimplices, (ii) order polytopes of finite partially ordered sets, and (iii) stable polytopes of perfect graphs. 


\section{Compressed $(0,1)$-Polytopes}

We fix integers $a_{i j}, b_{i}$ and $\varepsilon_{i}, 1 \leq i \leq m, 1 \leq j \leq n$, with each $\varepsilon_{i} \in\{0,1\}$, and suppose that the set of all solutions

$$
x=\left(x^{(1)}, x^{(2)}, \ldots, x^{(n)}\right) \in \mathbb{R}^{n}
$$

of the system of linear inequalities

$$
\begin{aligned}
b_{i} \leq \sum_{j=1}^{n} a_{i j} x^{(j)} \leq b_{i}+\varepsilon_{i}, & 1 \leq i \leq m, \\
0 \leq x^{(j)} \leq 1, & 1 \leq j \leq n,
\end{aligned}
$$

is nonempty. Thus the system of linear inequalities (1) and (2) determines a convex polytope $\mathcal{P} \subset \mathbb{R}^{n}$. The question whether $\mathcal{P}$ is a $(0,1)$-polytope or not seems to be rather difficult. However, once the convex polytope $\mathcal{P}$ turns out to be a $(0,1)$ polytope, we immediately conclude that $\mathcal{P}$ is compressed.

Theorem 1.1. Suppose that the convex polytope $\mathcal{P} \subset \mathbb{R}^{n}$ determined by the system of linear inequalities (1) and (2) is a $(0,1)$-polytope. Then $\mathcal{P}$ is compressed.

Our proof of Theorem 1.1 will be given in Section 2. The situation of Theorem 1.1 is particularly nice if the coefficient matrix $\left(a_{i j}\right)_{1 \leq i \leq m ; 1 \leq j \leq n}$ of the system of linear inequalities (1) is totally unimodular, because the required assumption that $\mathcal{P}$ is a $(0,1)$-polytope is automatically satisfied if $\left(a_{i j}\right)_{1<i<m ; 1<j<n}$ is totally unimodular.

A matrix is called totally unimodular if each of its subdeterminants belongs to $\{0,+1,-1\}$. It follows from Hoffman and Kruskal [HK] that if an $m \times n$ matrix $\left(a_{i j}\right)_{1 \leq i \leq m ; 1 \leq j \leq n}$ with each $a_{i j} \in \mathbb{Z}$ is totally unimodular, then, for arbitrary integers $b_{i}$ and $\varepsilon_{i}, 1 \leq i \leq m$, with each $\varepsilon_{i} \in\{0,1\}$, all of the vertices of the convex polytope in $\mathbb{R}^{n}$ determined by the system of linear inequalities (1) and (2) belong to $\mathbb{Z}^{n}$. Hence

Corollary 1.2. Let an $m \times n$ matrix $\left(a_{i j}\right)_{1 \leq i \leq m ; 1 \leq j \leq n}$ be totally unimodular. Then, for arbitrary integers $b_{i}$ and $\varepsilon_{i}, 1 \leq i \leq m$, with each $\varepsilon_{i} \in\{0,1\}$, the convex polytope determined by the system of linear inequalities (1) and (2) is compressed.

Examples of totally unimodular matrices contain vertex-edge incidence matrices of finite bipartite graphs. Consult, e.g., [Sch for the detailed information about totally unimodular matrices.

Example 1.3. (a) One of the most direct applications of Corollary 1.2 concerns the hypersimplex. Let $2 \leq d<n$ be integers. The $d$-th hypersimplex in $\mathbb{R}^{n}$ is the convex polytope $\Delta(n ; d) \subset \mathbb{R}^{n}$ which is the convex hull of all $(0,1)$-vectors $\left(x^{(1)}, x^{(2)}, \ldots, x^{(n)}\right)$ with $x^{(1)}+x^{(2)}+\cdots+x^{(n)}=d$. Since the $1 \times n$ matrix $[1,1, \ldots, 1]$ is totally unimodular, it follows that $\Delta(n ; d)$ is compressed.

(b) Let $P=\left\{\alpha_{1}, \alpha_{2}, \ldots, \alpha_{n}\right\}$ be a finite partially ordered set and $\mathcal{O}_{P}$ the order polytope [StaEC, Example 4.6.34] of $P$. Thus $\mathcal{O}_{P}$ is the convex polytope in $\mathbb{R}^{n}$ whose vertices are the $(0,1)$-vectors $\left(x^{(1)}, x^{(2)}, \ldots, x^{(n)}\right)$ satisfying that if $x^{(s)}=1$ and if $\alpha_{t} \leq \alpha_{s}$ in $P$, then $x^{(t)}=1$. In particular, the origin of $\mathbb{R}^{n}$ is a vertex of $\mathcal{O}_{P}$. Since the system of linear inequalities (i) $x^{(s)} \leq x^{(t)}$ for all $s$ and $t$ with $\alpha_{t} \leq \alpha_{s}$ and (ii) $0 \leq x^{(j)} \leq 1$ for all $1 \leq j \leq n$ determines $\mathcal{O}_{P}$, it follows from Theorem 1.1] that $\mathcal{O}_{P}$ is compressed.

(c) Let $G$ be a finite graph on the vertex set $V(G)=\{1,2, \ldots, n\}$ having no loops and no multiple edges, and $E(G)$ the edge set of $G$. We associate each subset 
$W \subset V(G)$ with the $(0,1)$-vector $\rho(W)=\sum_{j \in W} \mathbf{e}_{j} \in \mathbb{R}^{n}$. Here $\mathbf{e}_{j}$ is the $j$-th unit coordinate vector in $\mathbb{R}^{n}$. In particular, $\rho(\emptyset)$ is the origin of $\mathbb{R}^{n}$. A subset $W \subset V(G)$ is called stable if $\{i, j\} \notin E(G)$ for any $i, j \in W$ with $i \neq j$. Note that the empty set and all single-element subsets of $V(G)$ are stable. Let $S(G)$ denote the set of all stable sets of $G$. The stable polytope of $G$ is the $(0,1)$-polytope in $\mathbb{R}^{n}$ which is the convex hull of $\{\rho(W) ; W \in S(G)\}$. It then follows from Chvátal [Chv together with Theorem 1.1 that the stable polytope of $G$ is compressed if $G$ is a perfect graph. (A finite graph $G$ is called perfect if, for all induced subgraphs $H$ of $G$ including $G$ itself, the chromatic number of $H$ is equal to the maximal degree of complete subgraphs contained in $H$.)

\section{Proof of Theorem 1.1}

First of all, we prove Theorem 1.1 under the situation that each $\varepsilon_{i}=0$ in the system of linear inequalities (1). Let $a_{i j}$ and $b_{i}, 1 \leq i \leq m, 1 \leq j \leq n$, be integers, and consider the convex polytope determined by the system of linear equalities and linear inequalities

$$
\begin{gathered}
\sum_{j=1}^{n} a_{i j} x^{(j)}=b_{i}, \quad 1 \leq i \leq m, \\
0 \leq x^{(j)} \leq 1, \quad 1 \leq j \leq n .
\end{gathered}
$$

We say that, in general, an integral convex polytope $\mathcal{P} \subset \mathbb{R}^{n}$ possesses the integer decomposition property [Sch, p. 337] if, for all $k=1,2, \ldots$ and for all $\alpha \in k \mathcal{P} \cap \mathbb{Z}^{n}$, where $k \mathcal{P}=\{k \alpha ; \alpha \in \mathcal{P}\}$, there exist $\alpha_{1}, \alpha_{2}, \ldots, \alpha_{k} \in \mathcal{P} \cap \mathbb{Z}^{n}$ such that $\alpha=\alpha_{1}+\alpha_{2}+\cdots+\alpha_{k}$.

Lemma 2.1. If the convex polytope $\mathcal{P} \subset \mathbb{R}^{n}$ determined by the system of linear equalities (3) and linear inequalities (4) is a $(0,1)$-polytope, then $\mathcal{P}$ possesses the integer decomposition property.

Proof. Let $\delta_{1}, \delta_{2}, \ldots, \delta_{q}$ denote the vertices of $\mathcal{P} \subset \mathbb{R}^{n}$. If $\alpha \in k \mathcal{P} \cap \mathbb{Z}^{n}$, then

$$
\alpha=c_{1} \delta_{1}+c_{2} \delta_{2}+\cdots+c_{q} \delta_{q}
$$

with each $0 \leq c_{\ell} \in \mathbb{Q}$ and with $\sum_{\ell=1}^{q} c_{\ell}=k$. Let, say, $c_{1} \neq 0$. If $\delta_{1}^{(j)}=1$, then $\alpha^{(j)} \neq 0$. Hence $\alpha^{(j)} \geq 1$ since $0 \leq \alpha^{(j)} \in \mathbb{Z}$. If $\alpha^{(j)}=k$, then $\delta_{\ell}^{(j)}=1$ for all $1 \leq \ell \leq q$ with $c_{\ell} \neq 0$ since each $\delta_{\ell} \in\{0,1\}^{n}$ and $\sum_{\ell=1}^{q} c_{\ell}=k$. Hence $\beta=\alpha-\delta_{1} \in \mathbb{Z}^{n}$ satisfies

$$
\begin{array}{r}
\sum_{j=1}^{n} a_{i j} \beta^{(j)}=(k-1) b_{i}, \quad 1 \leq i \leq m, \\
0 \leq \beta^{(j)} \leq k-1, \quad 1 \leq j \leq n .
\end{array}
$$

Thus $\beta$ belongs to $(k-1) \mathcal{P} \cap \mathbb{Z}^{n}$. Apply induction on $k$, and we know

$$
\beta=\delta_{p_{1}}+\delta_{p_{2}}+\cdots+\delta_{p_{k-1}}
$$

for some $p_{1}, p_{2}, \ldots, p_{k-1} \in[q]$. (In general, we write $[q]$ for the $q$-element set $\{1,2, \ldots, q\}$.) Hence

$$
\alpha=\delta_{1}+\delta_{p_{1}}+\delta_{p_{2}}+\cdots+\delta_{p_{k-1}} .
$$

Thus $\mathcal{P}$ possesses the integer decomposition property, as required. 
Lemma 2.2. If the convex polytope $\mathcal{P} \subset \mathbb{R}^{n}$ determined by the system of linear equalities (3) and linear inequalities (4) is a $(0,1)$-polytope, then $\mathcal{P}$ is compressed.

Proof. A slight modification of the technique appearing in the proof of [Stu] Theorem 14.8] will be valid in our situation.

Let $\delta_{1}, \delta_{2}, \ldots, \delta_{q}$ denote the vertices of the $(0,1)$-polytope $\mathcal{P} \subset \mathbb{R}^{n}$. Fix any reverse lexicographic term order $<_{r e v}$ on the polynomial ring $K\left[y_{1}, y_{2}, \ldots, y_{q}\right]$ and write $I_{\mathcal{P}} \subset K\left[y_{1}, y_{2}, \ldots, y_{q}\right]$ for the toric ideal of $\mathcal{P} \subset \mathbb{R}^{n}$. Let

$$
f=\prod_{\ell=1}^{N} y_{t_{\ell}}-\prod_{\ell=1}^{N} y_{s_{\ell}}
$$

be an irreducible binomial belonging to $I_{\mathcal{P}}$ such that, say, $y_{s_{1}}$ is the smallest variable with respect to $<_{\text {rev }}$ among the variables appearing in $f$. We then have the relation

$$
\sum_{\ell=1}^{N} \delta_{t_{\ell}}=\sum_{\ell=1}^{N} \delta_{s_{\ell}}
$$

in $\mathbb{Z}^{n}$. Let $J \subset[n]$ denote the set of all $j$ with $\delta_{s_{1}}^{(j)}=1$ and $J^{\prime} \subset[n]$ the set of all $j^{\prime}$ with $\delta_{s_{1}}^{\left(j^{\prime}\right)}=0$. Thus $J \cup J^{\prime}=[n]$ and $J \cap J^{\prime}=\emptyset$.

If $j \in J$, then there is $1 \leq \ell_{j} \leq N$ with $\delta_{t_{\ell_{j}}}^{(j)}=1$. If $j^{\prime} \in J^{\prime}$, then there is $1 \leq \ell_{j^{\prime}} \leq N$ with $\delta_{t_{j_{j^{\prime}}}}^{\left(j^{\prime}\right)}=0$. Let $\left\{r_{1}, r_{2}, \ldots, r_{k}\right\} \subset[N]$ denote the set of those $r \in[N]$ such that either $\delta_{t_{r}}^{(j)}=1$ for some $j \in J$ or $\delta_{t_{r}}^{\left(j^{\prime}\right)}=0$ for some $j^{\prime} \in J^{\prime}$. Now,

$$
\alpha=\delta_{t_{r_{1}}}+\delta_{t_{r_{2}}}+\cdots+\delta_{t_{r_{k}}} \in k \mathcal{P} \cap \mathbb{Z}^{n}
$$

satisfies $\alpha^{(j)}>0$ for all $j \in J$ and $\alpha^{\left(j^{\prime}\right)}<k$ for all $j^{\prime} \in J^{\prime}$. Hence $\beta=\alpha-\delta_{s_{1}}$ satisfies

$$
\begin{aligned}
\sum_{j=1}^{n} a_{i j} \beta^{(j)}=(k-1) b_{i}, & 1 \leq i \leq m, \\
0 \leq \beta^{(j)} \leq k-1, & 1 \leq j \leq n .
\end{aligned}
$$

Thus $\beta \in(k-1) \mathcal{P} \cap \mathbb{Z}^{n}$. Since Lemma 2.1 guarantees that the polytope $\mathcal{P} \subset \mathbb{R}^{n}$ possesses the integer decomposition property,

$$
\beta=\delta_{p_{1}}+\delta_{p_{2}}+\cdots+\delta_{p_{k-1}}
$$

for some $p_{1}, p_{2}, \ldots, p_{k-1} \in[q]$. Then the binomial

$$
g=y_{t_{r_{1}}} y_{t_{r_{2}}} \cdots y_{t_{r_{k}}}-y_{s_{1}} y_{p_{1}} y_{p_{2}} \cdots y_{p_{k-1}}
$$

belongs to $I_{\mathcal{P}}$. Since $i n_{<_{\text {rev }}}(g)=y_{t_{r_{1}}} y_{t_{r_{2}}} \cdots y_{t_{r_{k}}}$ is squarefree and since $i n_{<_{\text {rev }}}(g)$ divides $i n_{<_{\text {rev }}}(f)=y_{t_{1}} y_{t_{2}} \cdots y_{t_{N}}$, it follows that the initial ideal $i n_{<_{\text {rev }}}\left(I_{\mathcal{P}}\right)$ of the toric ideal $I_{\mathcal{P}}$ with respect to $<_{\text {rev }}$ is squarefree, as desired.

We are now in the position to give a proof of Theorem[1.1. Let $\Phi: \mathbb{R}^{n} \rightarrow \mathbb{R}^{n+m}$ be the injective affine map defined by setting

$$
\Phi\left(x^{(1)}, \ldots, x^{(n)}\right)=\left(x^{(1)}, \ldots, x^{(n)}, \sum_{j=1}^{n} a_{1 j} x^{(j)}-b_{1}, \ldots, \sum_{j=1}^{n} a_{m j} x^{(j)}-b_{m}\right) .
$$


Then $\Phi\left(\mathbb{Z}^{n}\right) \subset \mathbb{Z}^{n+m}$. The convex polytope $\Phi(\mathcal{P}) \subset \mathbb{R}^{n+m}$ is a $(0,1)$-polytope which consists of all vectors $\left(z^{(1)}, \ldots, z^{(n)}, z^{(n+1)}, \ldots, z^{(n+m)}\right)$ satisfying

$$
\begin{array}{cc}
\sum_{j=1}^{n} a_{i j} z^{(j)}-z^{(n+i)}=b_{i}, & 1 \leq i \leq m, \\
0 \leq z^{(j)} \leq 1, \quad 0 \leq z^{(n+i)} \leq \varepsilon_{i}, & 1 \leq j \leq n, \quad 1 \leq i \leq m .
\end{array}
$$

Now, Lemma 2.2 guarantees that $\Phi(\mathcal{P})$ is compressed. Thus, by Lemma 2.3 below, the convex polytope $\mathcal{P}$ is compressed, as required.

Lemma 2.3. Let $\Phi: \mathbb{R}^{n} \rightarrow \mathbb{R}^{n^{\prime}}$ be an injective affine map with $\Phi\left(\mathbb{Z}^{n}\right) \subset \mathbb{Z}^{n^{\prime}}$ and $\mathcal{P} \subset \mathbb{R}^{n}$ an integral convex polytope. Then the toric ideal of $\mathcal{P}$ coincides with that of $\Phi(\mathcal{P}) \subset \mathbb{R}^{n^{\prime}}$.

Proof. Since $\Phi$ is an injective affine map, if $\delta_{1}, \delta_{2}, \ldots, \delta_{q}$ are the vertices of $\mathcal{P}$, then $\Phi\left(\delta_{1}\right), \Phi\left(\delta_{2}\right), \ldots, \Phi\left(\delta_{q}\right)$ are the vertices of $\Phi(\mathcal{P})$. As was especially noted in the second paragraph of Introduction, in the present paper, the toric ideal of an integral convex polytope is defined by using only the set of its vertices. Thus each of the toric ideals $I_{\mathcal{P}}$ and $I_{\Phi(\mathcal{P})}$ is a binomial ideal in the polynomial ring $K\left[y_{1}, y_{2}, \ldots, y_{q}\right]$ in $q$ variables. A binomial $f=\prod_{\ell=1}^{N} y_{t_{\ell}}-\prod_{\ell=1}^{N} y_{s_{\ell}}$ belongs to $I_{\mathcal{P}}$ if and only if $\left(\sum_{\ell=1}^{N} \delta_{t_{\ell}}\right) / N=\left(\sum_{\ell=1}^{N} \delta_{s_{\ell}}\right) / N$ in $\mathbb{Q}^{n}$, while $f=\prod_{\ell=1}^{N} y_{t_{\ell}}-\prod_{\ell=1}^{N} y_{s_{\ell}}$ belongs to $I_{\Phi(\mathcal{P})}$ if and only if $\left(\sum_{\ell=1}^{N} \Phi\left(\delta_{t_{\ell}}\right)\right) / N=\left(\sum_{\ell=1}^{N} \Phi\left(\delta_{s_{\ell}}\right)\right) / N$ in $\mathbb{Q}^{n^{\prime}}$. Since $\Phi$ is an affine map, $\left(\sum_{\ell=1}^{N} \Phi\left(\delta_{t_{\ell}}\right)\right) / N=\Phi\left(\left(\sum_{\ell=1}^{N} \delta_{t_{\ell}}\right) / N\right)$. Since $\Phi$ is injective, $\Phi\left(\left(\sum_{\ell=1}^{N} \delta_{t_{\ell}}\right) / N\right)=\Phi\left(\left(\sum_{\ell=1}^{N} \delta_{s_{\ell}}\right) / N\right)$ if and only if $\left(\sum_{\ell=1}^{N} \delta_{t_{\ell}}\right) / N=\left(\sum_{\ell=1}^{N} \delta_{s_{\ell}}\right) / N$. Thus $f \in I_{\mathcal{P}}$ if and only if $f \in I_{\Phi(\mathcal{P})}$. Hence $I_{\mathcal{P}}=I_{\Phi(\mathcal{P})}$, as desired.

\section{REFERENCES}

[Chv] V. Chvátal, On certain polytopes associated with graphs, J. Combin. Theory (B) 18 (1975), 138 - 154. MR 51:7949

[HK] A. Hoffman and J. Kruskal, Integral boundary points of convex polyhedra, in "Linear Inequalities and Related Systems" (H. Kuhn and A. Tucker, Eds.), Princeton University Press, Princeton, NJ, 1956, pp. 223 - 246. MR 18:980b

[OhHerHi] H. Ohsugi, J. Herzog and T. Hibi, Combinatorial pure subrings, Osaka J. Math. 37 (2000), 745-757.

[Sch] A. Schrijver, "Theory of Linear and Integer Programming," John Wiley \& Sons, New York, 1986. MR 88m:90090

[Sta] R. Stanley, Decompositions of rational convex polytopes, Ann. Discrete Math. 6 (1980), 333 - 342. MR 82a:52007

[StaEC] R. Stanley, "Enumerative Combinatorics, Volume I," Wadsworth \& Brooks/Cole, Pacific Grove, Calif., 1986. MR 87j:05003

[Stu] B. Sturmfels, "Gröbner Bases and Convex Polytopes," Amer. Math. Soc., Providence, RI, 1995. MR 97b:13034

Department of Mathematics, Graduate School of Science, Osaka University, ToyONAKA, OSAKA 560-0043, JAPAN

E-mail address: ohsugi@math.sci.osaka-u.ac.jp

Department of Mathematics, Graduate School of Science, Osaka University, ToyONAKA, OSAKA 560-0043, JAPAN

E-mail address: hibi@math.sci.osaka-u.ac.jp 